\title{
Real-Time Human Cross-Race Aging-Related Face Appearance Detection with Deep Convolution Architecture
}

DOI:

10.1007/s11554-019-00903-9

\section{Document Version}

Accepted author manuscript

Link to publication record in Manchester Research Explorer

\section{Citation for published version (APA):}

Tian, Q., Zhang, W., Mao, J., \& Yin, H. (2019). Real-Time Human Cross-Race Aging-Related Face Appearance Detection with Deep Convolution Architecture. Journal of Real-Time Image Processing.

https://doi.org/10.1007/s11554-019-00903-9

\section{Published in:}

Journal of Real-Time Image Processing

\section{Citing this paper}

Please note that where the full-text provided on Manchester Research Explorer is the Author Accepted Manuscript or Proof version this may differ from the final Published version. If citing, it is advised that you check and use the publisher's definitive version.

\section{General rights}

Copyright and moral rights for the publications made accessible in the Research Explorer are retained by the authors and/or other copyright owners and it is a condition of accessing publications that users recognise and abide by the legal requirements associated with these rights.

\section{Takedown policy}

If you believe that this document breaches copyright please refer to the University of Manchester's Takedown Procedures [http://man.ac.uk/04Y6Bo] or contact uml.scholarlycommunications@manchester.ac.uk providing relevant details, so we can investigate your claim.

\section{OPEN ACCESS}




\title{
Real-Time Human Cross-Race Aging-Related Face Appearance Detection With Deep Convolution Architecture
}

\author{
Qing Tian ${ }^{\mathrm{a}, 1,2,3,4}$, Wenqiang Zhang ${ }^{\mathrm{b}, 1}$, Junxiang Mao ${ }^{\mathrm{c}, 1}$, Hujun Yin ${ }^{\mathrm{d}, 5}$ \\ ${ }^{1}$ School of Computer and Software, Nanjing University of Information Science and Technology, Nanjing, China \\ ${ }^{2}$ Collaborative Innovation Center of Atmospheric Environment and Equipment Technology, Nanjing University of Information Science and \\ Technology, Nanjing, China \\ ${ }^{3}$ Jiangsu Engineering Center of Network Monitoring, Nanjing University of Information Science and Technology, Nanjing, China \\ ${ }^{4}$ MIIT Key Laboratory of Pattern Analysis and Machine Intelligence, Nanjing University of Aeronautics and Astronautics, Nanjing, China \\ ${ }^{5}$ School of Electrical and Electronic Engineering, The University of Manchester, Manchester, UK
}

Received: 6 May, 2019 / Accepted: date

\begin{abstract}
Human age estimation (AE) is an emerging research topic in computer vision and machine learning and has attracted increasing amount of research due its wide potential applications. In the process of human aging, facial appearances change from glabrous to crinkly similarly across all races, from European, Hispanic and African to Asian. To specially explore the relationships between aging and facial appearances across races, this paper is devoted to determining the correspondence between facial aging and facial appearances. Specifically, we first extract appearance vector features from facial images with their spatial structure preserved. Then, we propose to select the aging-related features shared by different races to explore their agingrelated common facial regions, while removing redundant features. Thirdly, we improve the proposed model by incorporating potential cross-race relationships in an automated learning manner. Additionally, we extend our model with deep convolution architecture. Finally, we evaluate the proposed methodologies on a large face aging database with real-time efficiency.
\end{abstract}

\section{Background}

Facial age estimation (AE) or aging research is an interesting research topic and has attracted increased attention in computer vision and patter recognition because of its useful applications such as security monitoring ([28]), consumer recommendation ([18]), identity recognition ([26], [3]) and information forensics ([6], [53]).

To estimate facial appearance age, various methods have been presented. They generally fall in three groups, namely,

\footnotetext{
${ }^{a}$ E-mail: tianqing@nuist.edu.cn [corresponding author]

${ }^{b}$ E-mail: cnzhangwenqiang@gmail.com

cE-mail: 20161308081@nuist.edu.cn

dE-mail: hujun.yin@manchester.ac.uk
}

classification-based (e.g. [1, 8, 10, 19, 36, 43]), regressionbased (e.g. [5, 7, 9, 20-22, 27, 39, 40, 42, 46, 48, 49]), and hybrid approaches (e.g. $[11,12,17])$. When we treat each age as an individual class, AE can be formulated as classification learning. To this end, [19] first extracted the Active Appearance Models from face images and then directly used the nearest neighbor or neural networks to classify facial ages. [43] developed a Gaussian mixture model to roughly classify human faces into several age bands. Additionally, [8] generated conditional probability neural network by taking into account the distributions of the aging sequence to estimate human age. To preserve the similarity of adjacent ages, [1] encoded the age labels as soft probability distributions. More recently, [36] adopted the extreme learning machines for age classification. Motivated by the wide success of deep learning (DL) [45, 52], the DL-based models have also be used to classify human age ([31], [50], [47], [24]).

In accordance with the continuity of human aging, the AE problem should be treated as regression rather than classification. Along this line, [20] regressed human facial ages using a quadratic fitting function. [37] constructed a statistically linear model for age regression. [7] adopted a multilinear regressor to fit ages. Impressed by the promising performance of $\xi$-SVR, [27] employed it for human age regression. Furthermore, [49] modelled a SDP function for robust age estimation. Later, [48] further speeded up and optimized their regressor, instead of relying on the EM algorithm. Besides, [9] constructed the called AGES regressor to recover missing age patterns outside the gallery. Although the aforementioned methods can achieve the goal of age regression to certain extent, the ordinal characteristic of aging sequence is not utilized $([5,42])$. To alleviate this issue, [5] constructed an ordinal model (OHRank) to regress human ages. In addition, [21, 23] constructed an ordinal distance space to train more discriminative age regressor. Then, [22] intro- 
duced age-related feature selection to generalize the ability of the regressor.

However, the methods reviewed above treat AE separately from other facial attributes, which hinders performance of AE, as some research (e.g. [13], [14]) showed that facial appearances are changing gradually with increasing wrinkles in the process of human aging and that different race people are aging similarly in facial appearance. To this end, in this paper we concentrate on exploring facial aging-related appearance features across races. Specially, we explore which regions of human facial appearance are more relevant to their aging, and incorporate jointly the feature selection and facial appearance structure preservation to improve the accuracy of AE. Additionally, we extend the model with deep convolution architecture to improve its discriminating capacity with real-time efficiency. To the best of our knowledge, little research has been dedicated to this problem.

The rest of this paper is organized as follows. Section 2 presents our proposed method. Section 3 reports validation results and provides an analysis. Finally, Section 4 concludes this work.

\section{The proposed method}

Related literature ([13], [14]) shows that facial appearances change gradually with increasing wrinkles in the process of human aging, and that different races age similarly in facial appearance. Herein we explore aging-related facial appearance regions and thus adopt these features to benefit AE, removing aging-irrelevant facial features, in order to improve the discriminative and generalization ability of the AE estimators.

\subsection{Objective function}

To find out facial appearance features related to aging, without loss of generality, we take the ridge regression (RR) [15] as the base model for facial age regression. Besides, assume that we are given $N n_{1}$-by- $n_{2}$ facial images with centralized vector-form feature representations and age labels $\left\{x_{i}, y_{i}\right\}_{i=1}^{N} \in R^{d} \times R$ (i.e., $\left.n_{1} \times n_{2}=d\right)$. Then, we can train an $\mathrm{RR}$ regressor on these images by minimizing the following function:

$$
\min _{w} \frac{1}{N} \sum_{i=1}^{N}\left\|w^{T} x_{i}-y_{i}\right\|_{2}^{2}+\lambda\|w\|_{2}^{2},
$$

where $w \in \mathbb{R}^{d}$ is the regression vector and $\lambda$ stands for the nonnegative hyper-parameter. While $x \in \mathbb{R}^{d}$ is a vector representation reshaped from the $i$ th image matrix, the spatial structure of the original image is not specifically preserved in the vectorization transformation that the spatial relationships of the image, such as the neighbor-similarity, are not considered. To alleviate such drawbacks, we need to incorporate the omitted spatial information for the RR-based image regression. Motivated by our previous work [41], the spatial smooth subspace learning (SSSL) [4] can be used desirably to compensate such missed image spatial information. In SSSL [4], the spatial information in the images is made for use by spatially smoothing the projection vector $w$ as

$\Gamma=\|\Delta w\|^{2}=w^{T} \Delta^{T} \Delta w=w^{T} \Upsilon w$

with

$\Delta=D_{1} \otimes I_{2}+D_{2} \otimes I_{1}$

where $\otimes$ denotes the Kronecker operator, $\left\{I_{j}\right\}_{j=1}^{2}$ are $n_{j^{-}}$ order identity matrices, and the smoothing operator $D_{j} \in$ $\mathbb{R}^{d \times d}$ is a block-diagonal matrix with diagonal elements $\{-1$, $-2, \ldots .,-2,-1\}$ and sub-diagonal elements of all ones. Replacing the second term of (1) by (2) yields the SSSL regularized RR (shortened as RR-SSSL)

$$
\min _{w} \frac{1}{N} \sum_{i=1}^{N}\left\|w^{T} x_{i}-y_{i}\right\|_{2}^{2}+\frac{\lambda}{2} w^{T} \Upsilon w .
$$

While the spatial structure information of facial images is incorporated in Eq.(4), the resulting RR regressor in real applications tends to over-fitting since $d=n_{1} \times n_{2}$ is usually much higher than image numbers $N$, particularly in the scenario of AE. Moreover, the features of facial appearance suffer from serious redundance. Therefore, it is desirable to perform feature selections for the RR-SSSL regressor. In addition, research ([13], [14]) shows that people of different races are aging similarly in facial appearance. Motivated by the above findings, we propose to perform similar features selection across races on RR-SSSL via group-lasso algorithm ([29]), termed as RR-SSSL-GL, with remodelled objective function as

$$
\begin{gathered}
\min _{W=\left[w_{1}, \ldots, w_{R}\right]} \sum_{r=1}^{R} \frac{1}{N_{r}} \sum_{i=1}^{N_{r}}\left\|w_{r}^{T} x_{r i}-y_{r i}\right\|_{2}^{2}+\frac{\lambda_{1}}{2} \operatorname{tr}\left(W^{T} r W\right) \\
+\frac{\lambda_{2}}{2}\|W\|_{2,1},
\end{gathered}
$$

where $R$ denotes the number of human races, $x_{r i}$ and $y_{r i}$ respectively denote the $i$ th instance and its label of $N_{r}$ samples from the $r$ th race, $\operatorname{tr}(\cdot)$ stands for the trace operator, and $W=\left[w_{1}, \ldots, w_{R}\right] \in \mathbb{R}^{d \times R}$ is the entire projection matrix for the races. The last $l_{2 / 1}$-term in (5) helps select these facial features that are most relevant to aging and shared by all races.

Besides the joint feature selection characterized by $l_{2 / 1^{-}}$ term in (5), there possibly exist potential correlations between the races in the process of learning their joint projection matrix $W$, which are expected to be incorporated in the objective function. Motivated by the work of [51] and [25], 
we propose to model these potential correlations among the projection vectors $w_{i}(i=1, \ldots, R)$ of $W$ in an automated learning manner. To be specific, we introduce the covariance matrix (denoted as $\Omega$ ) of $W$ to model the possible relationships within and between $w_{i}, i=1, \ldots, R$. Along this line, (5) can be remodelled as

$$
\begin{gathered}
\min _{\left\{W=\left[w_{1}, \ldots, w_{R}\right], \Omega\right\}} \sum_{r=1}^{R} \frac{1}{N_{r}} \sum_{i=1}^{N_{r}}\left\|w_{r}^{T} x_{r i}-y_{r i}\right\|_{2}^{2}+\frac{\lambda_{1}}{2} \operatorname{tr}\left(W^{T} \Upsilon W\right) \\
+\frac{\lambda_{2}}{2}\|W\|_{2,1}+\frac{\lambda_{3}}{2} \operatorname{tr}\left(W \Omega^{-1} W^{T}\right), \\
\text { s.t. } \quad \Omega \succeq 0 \\
\operatorname{tr}(\Omega)=1,
\end{gathered}
$$

where $\Omega \succeq 0$ guarantees the positive semi-definiteness of $\Omega$ and $\operatorname{tr}(\Omega)=1$ controls its complexity, $\lambda_{1}, \lambda_{2}$ and $\lambda_{3}$ are nonnegative trade-off parameters. The model of (6) is termed as RR-SSSL-GL-O for short. An illustration of this methodology is shown in Figure 1.

\subsection{Optimization}

Since (6) is jointly convex with respect to $W$ and $\Omega$, we take an alternating strategy to optimize it. That is, we fix one variable and optimize with regard to another.

When $\Omega$ is fixed, then (6) can be equivalently rewritten as

$$
\begin{gathered}
\min _{W=\left[w_{1}, \ldots, w_{R}\right]} \sum_{r=1}^{R} \frac{1}{N_{r}} \sum_{i=1}^{N_{r}}\left\|w_{r}^{T} x_{r i}-y_{r i}\right\|_{2}^{2}+\frac{\lambda_{1}}{2} \operatorname{tr}\left(W^{T} \Upsilon W\right) \\
+\frac{\lambda_{2}}{2}\|W\|_{2,1}+\frac{\lambda_{3}}{2} \operatorname{tr}\left(W \Omega^{-1} W^{T}\right),
\end{gathered}
$$

which is convex but not generally smooth because of the non-smooth third term. To solve (7), we adopt an accelerated proximal gradient (APG) algorithm [2]. Specifically, (7) can be grouped into two parts, i.e., smooth part $f(W):=$ $\sum_{r=1}^{R} \frac{1}{N_{r}} \sum_{i=1}^{N_{r}}\left\|w_{r}^{T} x_{r i}-y_{r i}\right\|_{2}^{2}+\frac{\lambda_{1}}{2} \operatorname{tr}\left(W^{T} \Upsilon W\right)+\frac{\lambda_{3}}{2} \operatorname{tr}\left(W \Omega^{-1} W^{T}\right)$ and non-smooth term $g(W):=\frac{\lambda_{2}}{2}\|W\|_{2,1}$. Then, we define the generalized gradient update rule as follows

$$
\begin{aligned}
Q\left(W, W^{t}\right) & =f\left(W^{t}\right)+<W-W^{t}, \nabla f\left(W^{t}\right)> \\
& +\frac{l}{2}\left\|W-W^{t}\right\|_{F}^{2}+g(W),
\end{aligned}
$$

where $\|\cdot\|$ denotes the Frobenius norm operator, $\nabla f\left(W^{t}\right)$ is the gradient of $f(W)$ with regard to $W$ at the $t$ th iteration, $l$ is the step size obtained through the line search techniques [44], and $\langle\cdot, \cdot\rangle$ is the matrix inner-product operator. Then,
$W^{t+1}$ at the $(t+1)$ th step can be updated as

$$
\begin{aligned}
& W^{t+1}=\arg \min _{W} \frac{1}{2}\left\|W-\left(W^{t}-\frac{1}{l} \nabla f\left(W^{t}\right)\right)\right\|_{F}^{2}+\frac{1}{l} g(W) \\
& =\arg \min _{W=\left[W_{1:}^{T}, \ldots, W_{d:}^{T}\right]^{T}} \frac{1}{2} \sum_{j=1}^{d}\left\|W_{j:}-\left(W^{t}-\frac{1}{l} \nabla f\left(W^{t}\right)\right)_{j:}\right\|_{F}^{2} \\
& +\frac{\lambda_{2}}{2 l}\left\|W_{j:}\right\|_{2} \text {, }
\end{aligned}
$$

where $W_{j}$ indicates the $j$ th row of $W$. From (9), it can be seen that the entire $W$ can be solved in a divide-and-conquer manner and enjoys the following analytical solution [2]

$W_{j:}^{t+1}=\left\{\begin{array}{l}\left(\begin{array}{l}\left.1-\frac{\lambda_{2}}{2 l\left\|\left(W^{t}-\frac{1}{l} \nabla f\left(W^{t}\right)\right)_{j:}\right\|_{2}}\right) \\ \quad \text { if }\left\|\left(W_{t}-\frac{1}{l} \nabla f\left(W^{t}\right)\right)_{j:}\right\|_{2}>\frac{\lambda_{2}}{2 l}\end{array}\right. \\ 0, \quad \text { otherwise. }\end{array}\right.$

Through iteratively performing (10) until $Q\left(W, W^{t}\right)$ converges to $f(W)+g(W)$, the final solution $W^{*}$ is obtained.

When $W$ is fixed, then (6) equivalently becomes

$$
\begin{gathered}
\min _{\Omega} \operatorname{tr}\left(W \Omega^{-1} W^{T}\right) \\
\text { s.t. } \quad \Omega \succeq 0, \\
\operatorname{tr}(\Omega)=1 .
\end{gathered}
$$

Interestingly, (11) also has analytical solution

$\Omega=\frac{\left(W^{T} W\right)^{\frac{1}{2}}}{\operatorname{tr}\left(\left(W^{T} W\right)^{\frac{1}{2}}\right)}$.

For initialization, we set $\Omega$ to $\frac{1}{R} I_{R}$ with $I_{R}$ being an $R$-order identity matrix. We optimize (6) with regard to $W$ and $\Omega$ in an alternating manner until convergence. The complete algorithm are summarized in Algorithm 1. It is worth noting that since the methods RR, RR-SSSL, RR-SSSL-GL presented above are covered by RR-SSSL-GL-O, so they also can be solved via the same algorithm, or directly with the analytical solutions for RR and RR-SSSL (due to space limit, we omit the details).

\subsection{Time complexity analysis}

The time complexity of Algorithm 1 is mainly in computing the gradient of $f(W)$ and optimizing for (9). More specifically, the time complexity for calculating the gradient of $f(W)$ is $\mathscr{O}(d N)$, and the time complexity for solving (9) is $\mathscr{O}(d R)$. Therefore, the total time complexity of Algorithm 1 is $\mathscr{O}\left(d(N+R) I_{\text {iter }}\right)$. 

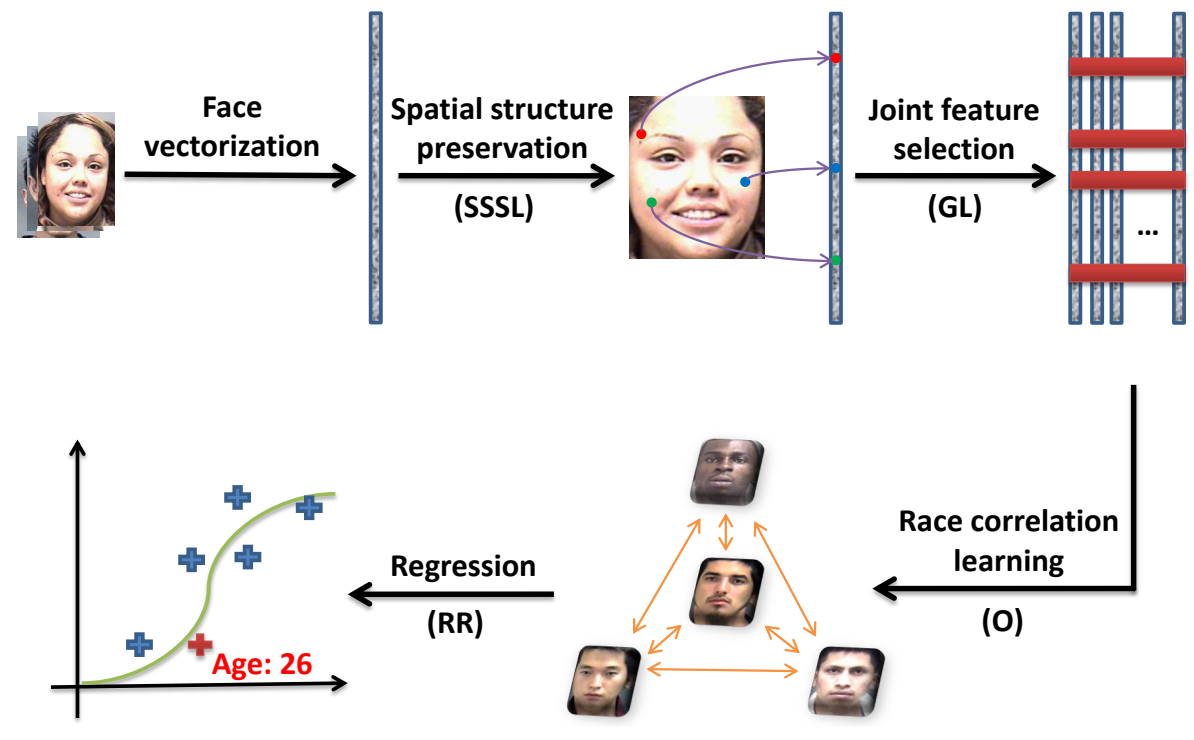

Fig. 1 Methodology illustration of the proposed RR-SSSL-GL-O method on face image.

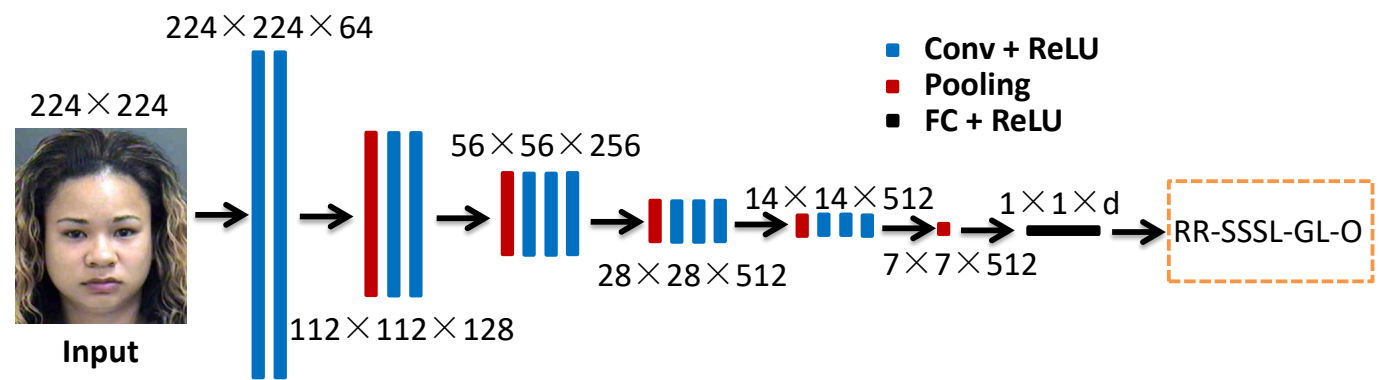

Fig. 2 Architecture of our RR-SSSL-GL-O model extended with deep convolution networks. 'Conv' denotes the convolution layers, 'ReLU' is the activation function, 'Pooling' denotes the max-pooling layers, and 'FC' indicates the fully-connected layers.

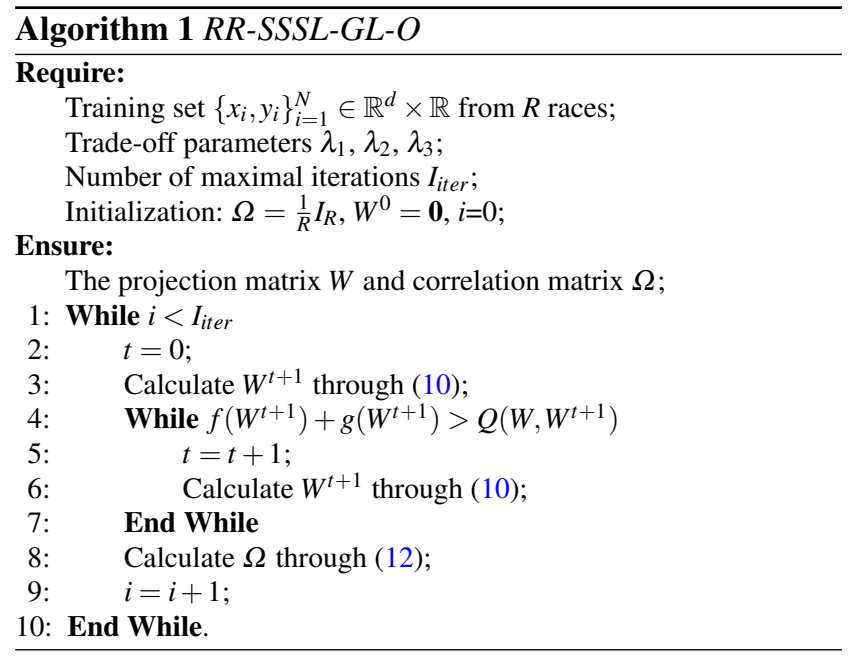

\subsection{Extension with deep architecture}

Motivated by the wide success of deep convolution learning in the field of image processing, we further extend our model with deep architecture. Along this line, we refer to the VG-
G network [38] and modify it to cater for our requirements. Specially, we modify the last fully-connected layer with $d$ neurons and take its output to replace the hand-crafted features for the proposed RR-SSSL-GL-O model. In this way, the architecture of our model is deepened with deep feature representations that are learnt automatically from training data. The complete architecture of our deep model is illustrated in Figure 2.

\section{Experiments}

To evaluate the efficiency of the proposed methodology in discovering aging-related facial appearances for AE across races, we have conducted experiments on the largest publicly available human aging face dataset, namely Morph (Album II) [35], in which each image is annotated with corresponding age and race information. In addition, The Morph dataset contains over 55,000 unique images of more than 13,000 individuals, spanning from 2003 to late 2007. The ages range from 16 to 77 with a median age of 33 . The faces were drawn from European, Hispanic, African, Asian, 
and Other races. Examples of the Morph faces are shown in Figure 3. The distributions of face samples are summa-
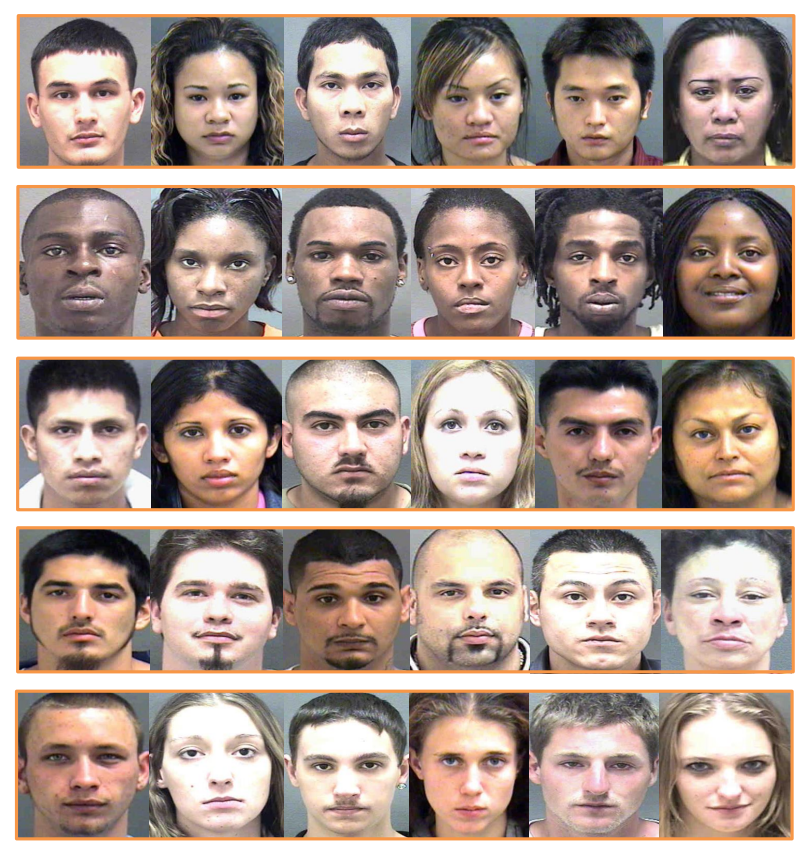

(b)

Fig. 3 Face image examples of Asian (a), African (b), Hispanic (c), Other (d) and European (e) from the Morph database.

rized in Table 1. It can be seen that samples distributions are not balanced in terms of ages and races. We cropped

Table 1 Races and ages distributions of the Morph database.

\begin{tabular}{|c|c|c|c|c|c|c|}
\hline Race & European & Other & Hispanic & African & Asian & Total \\
\hline Total & 10559 & 63 & 1769 & 42589 & 154 & 55134 \\
\hline & & & & & & \\
\hline Age & $<20$ & $20-29$ & $30-39$ & $40-49$ & $50+$ & Total \\
\hline Total & 7469 & 16325 & 15357 & 12050 & 3933 & 55134 \\
\hline
\end{tabular}

the face regions from the face images according to eyes locations, normalized them to $50 \times 50$ pixels and then pulled them into 2500-dimensional vectors as representation features. We tuned the trade-off parameters through a five-fold cross-validation in range $\left[0,10^{-5}, 10^{-3}, 10^{-1}, 10^{1}, 10^{3}\right.$, $10^{5} \mathrm{]}$. The results reported are the averages over ten runs with random data partitions. In the experiments, the methods to be compared are summarized below

- RR: The method formulated in (1).

- RR-SSSL: The method formulated in (4).

- RR-GL: The method formulated in (6) with removing $\Omega$ and setting $\lambda_{1}=0$ and $\lambda_{3}=0$.

- RR-SSSL-GL: The method formulated in (5).

- RR-SSSL-GL-O: The method formulated in (6). (d)
- Deep-RR-SSSL-GL-O: The extended counterpart of RRSSSL-GL-O with deep convolution architecture shown in Figure 2. In the experiment, the model was pretrained on the AgeDB [30] dataset and then fine-tuned on Morph with certain percentage of training samples. The model parameters were optimized through stochastic gradient descent (SGD) [16] with batch-size of 64, initial learning rate of 0.001 (which was decayed by 0.1 per 15 epoches), momentum of 0.9 , weight decay of 0.0005 , and last fully connected layers with dropout ratio of 0.5 .

\subsection{Performance comparison}

Experimental comparisons were performed on pixel features extracted from the Morph database. The results are shown in Figure 4. We have the following findings. Firstly, with easing percentage of tra 1 methods are reduced. It validates that increasing training percentage produces better discriminative ability of the AE estimators. Secondly, the MAEs of AE yielded by RR, RRSSSL, RR-SSSL-GL, and RR-SSSL-GL-O reduce successively, demonstrating the rationality and effectiveness of $\mathrm{p}$ reserving spatial structure (SSSL), selecting jointly discriminative features (GL) and incorporating joint correlation relationships between the races. Thirdly, the MAEs by RR and RR-SSSL (both them are not incorporated with join$t$ feature selection) are significantly higher than the other methods with joint feature selection, indicating the benefit of removing redundant features to reduce the dimensionality of data. Fourthly, the MAEs on different races are not at the same level. Generally, the Hispanic race has the lowest MAEs, while the Other race has the highest MAEs. From Table 1, we can see that the Other race possesses very few samples, which may seriously limit the AE generalization performance. By contrast, although the number of samples in Hispanic race is fairly limited (especially compared with African and European), the lowest MAEs on Hispanic demonstrate its best discriminative characteristic in facial aging. Last but not least, the MAEs yielded by the Deep-RR-SSSLGL-O are significantly the lowest. It demonstrates that extending the model architecture with deep layers of convolution networks can significantly improve its predicting accuracy on facial age.

\subsection{Aging-related facial appearance exploration}

To explore which regions the degree of facial appearance are changing relating to human aging, we reshape the learned projection vectors $w_{i}(i=1, \ldots R)$ corresponding to the races (with 30 percentage of samples for training) and visualize them in Figure 5. We can observe the following rules. Firstly, compared with the baseline method $R R$, although the face 


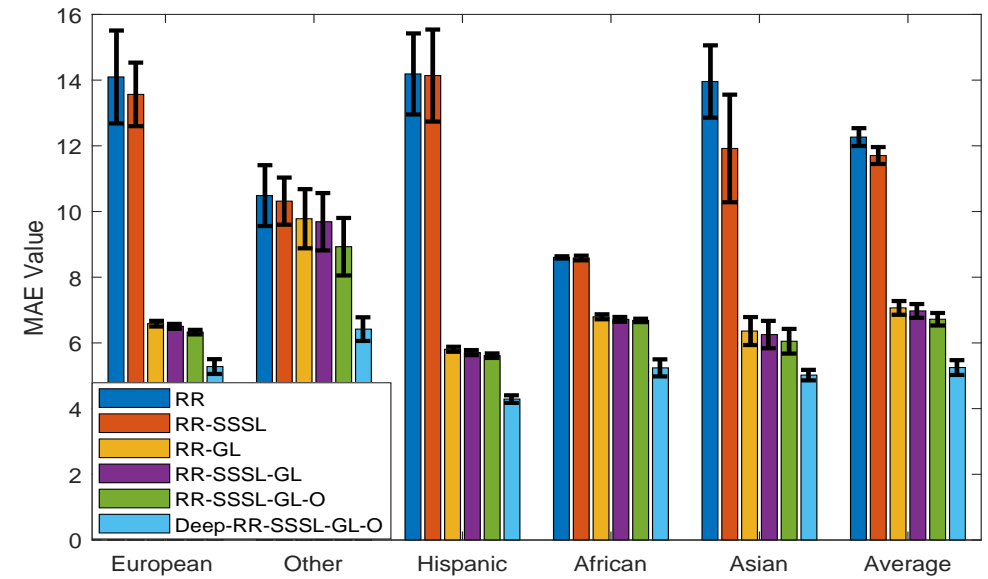

(a) 10 percent for training

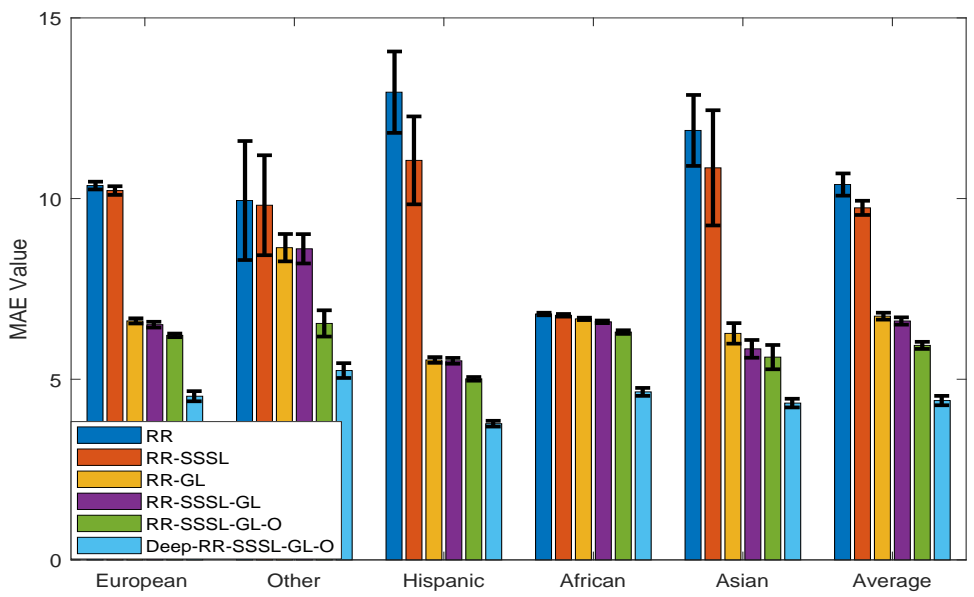

(b) 30 percent for training

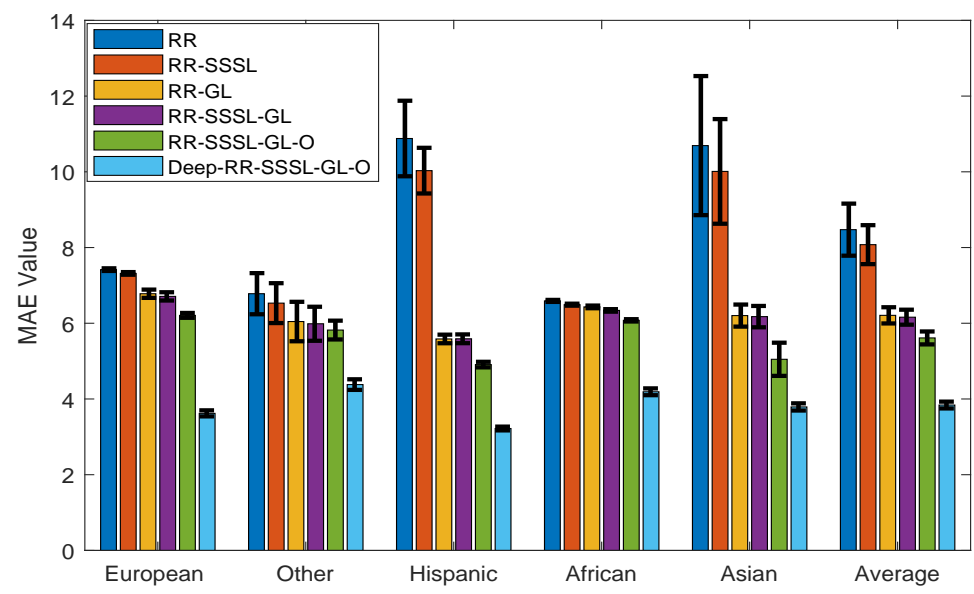

(c) 50 percent for training

Fig. 4 Age estimation performance comparison with 10 percent (a), 30 percent (b), and 50 percent (c) of samples for training while the rest for testing on the Morph database.

spatial structure information is preserved via SSSL technology in $R R$-SSSL, the learned aging-related facial appearances are still not obvious. The benefit of SSSL is destroyed by the high-dimensional facial appearance representations (with 2500-dimensional pixel-features but relatively small number of training samples). Secondly, from RR-GL to RR- 

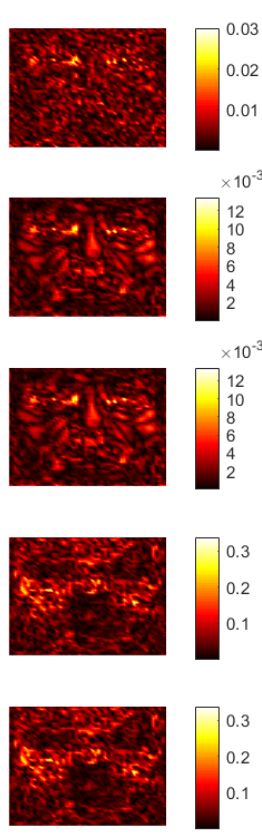
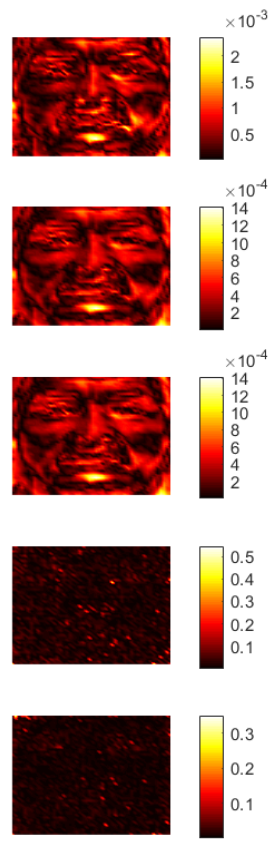
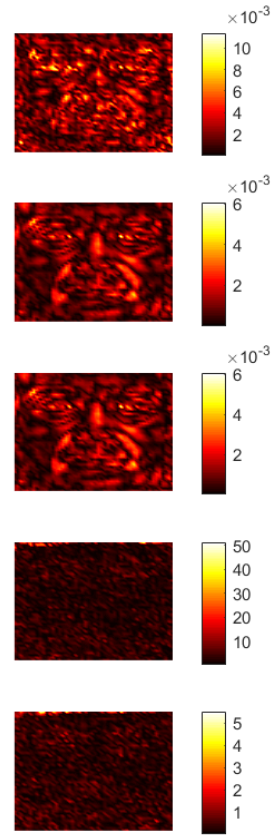
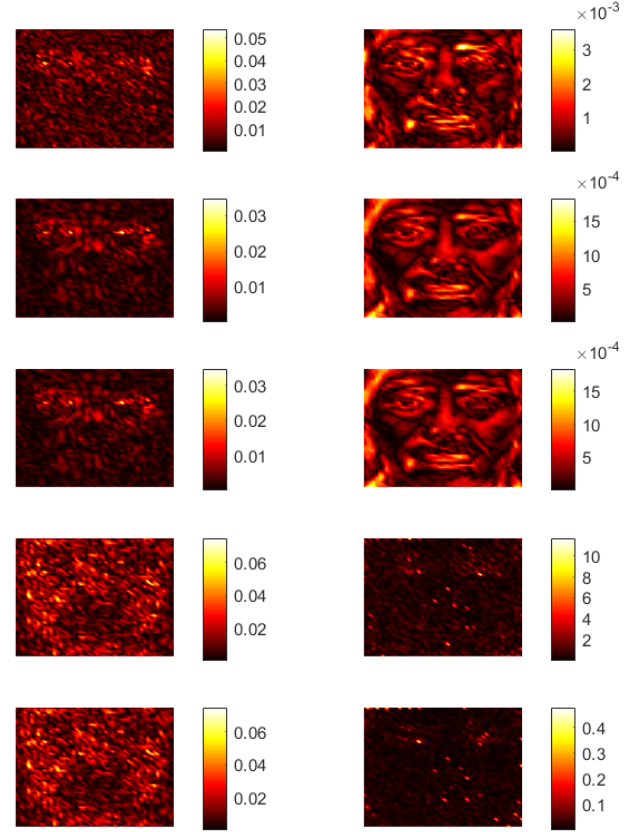

Fig. 5 2D-visualization of the learned projection vectors regarding the face images of European, Other, Hispanic, African and Asian races (from the first column to the last column) by RR-SSSL-GL-O, RR-SSSL-GL, RR-GL, RR-SSSL, and RR (from the first row to the last row).

SSSL-GL and to RR-SSSL-GL-O, the facial appearance regions learned related to human aging are gradually becoming refined with clear contours. It demonstrates that incorporating more reasonable prior knowledge helps discover better facial aging-related appearance regions. Thirdly, the amplitudes of learned features regarding to different facial regions are almost the same. Generally, the regions around eyes, cheekbone and mouth are with high amplitudes, showing their great relevance to facial aging. Last but not least, the facial feature contours with respect to the Asian and Other races are more clear to distinguish than the other races. As previously analyzed, the Asian and Other races possess fewer face samples than the other three races. It means that compared to the other races, the Asian and Other races suffer from smaller facial appearance variances in head pose, illumination, and facial expressions, etc, which hinder discovering the contours of facial aging-related appearances.

\subsection{Correlations between the races in facial aging}

To further explore the correlation relationships among the races, we visualize the learned covariance matrix $\Omega$ among the projection vectors regarding the races in Figure 6 . We can see that the degrees of correlations between the races are not the same, meaning that correlation relationships of the races are not the same and should be learned naturally from data instead of defining arbitrarily.

\subsection{Real-time verification}

In order to evaluate the real-time performance of the compared methods, we conducted experiments on the Morph dataset on Matlab R2017b computing station with i7-CPU and 48GB RAM memory. Specially, we randomly selected $10 \%$ instances as training set and took the rest as testing set. For statistical reliability, we repeated five times and report the averaged time cost for training and testing stage in Table 2 .

From the reported results, we can see that nearly all the models can be trained with real-time cost (in an off-line manner), especially for testing, all the methods can make decisions less than 1 millisecond. They illustrate the real-time efficiency of all the compared methods.

\section{Conclusion}

In this work, we mainly explored the relations between human aging and relevant facial appearances shared by different races. We first extracted appearance vector features from facial images with spatial structure preservation. Then, we proposed to select aging-related features shared by different races in a joint manner to explore their aging-related common facial regions, while removing the redundant features. Thirdly, we improved the proposed model by incorporating the potential cross-race relationships in automated learning manner. In addition, we further extended our model with deep convolution networks for more discriminative fea- 


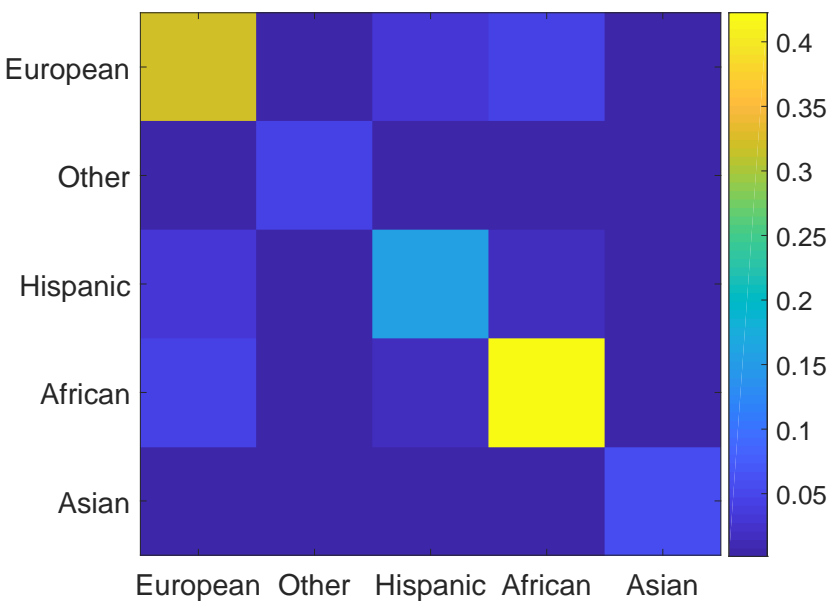

Fig. 6 Visualization of the learned covariance matrix $\Omega$ among the races on the Morph database.

Table 2 Averaged real-time performance of the compared methods (in seconds per image). The Deep-RR-SSSL-GL-O model was trained in an off-line manner, so we do not count its training time cost.

\begin{tabular}{|c||c|c|c|c|c|c|}
\hline Method & $R R$ & $R R$-SSSL & $R R$-GL & $R R$-SSSL-GL & RR-SSSL-GL-O & Deep-RR-SSSL-GL-O \\
\hline Training time & 0.0011 & 0.0013 & 0.3399 & 0.5984 & 1.9183 & $/$ \\
\hline Testing time & 0.0003 & 0.0003 & 0.0004 & 0.0005 & 0.0004 & 0.0754 \\
\hline
\end{tabular}

ture representations. Finally, we evaluated the performance superiority and real-time efficiency of the proposed methodologies on large-scale face data set. In future, we will perfor$m$ deep research with distributed data scenarios with privacy preservation [32-34].

\section{Acknowledgments}

This work was supported partially by the National Natural Science Foundation of China under grant 61702273, the Natural Science Foundation of Jiangsu Province under grant BK20170956, the Natural Science Foundation of the Jiangsu Higher Education Institutions of China under grant 17KJB520022, the Fundamental Research Funds for the Central Universities No. NJ2019010, the Project Funded by the Priority Academic Program Development of Jiangsu Higher Education Institutions, the Priority Academic Program Developmen$\mathrm{t}$ of Jiangsu Higher Education Institutions (PAPD) fund, and the Startup Foundation for Talents of Nanjing University of Information Science and Technology.

\section{References}

1. Fares Alnajar, Caifeng Shan, Theo Gevers, and JanMark Geusebroek. Learning-based encoding with soft assignment for age estimation under unconstrained imaging conditions. Image and Vision Computing, 30(12):946-953, 2012.
2. Amir Beck and Marc Teboulle. A fast iterative shrinkage-thresholding algorithm for linear inverse problems. Siam Journal on Imaging Sciences, 2(1):183-202, 2009.

3. Simone Bianco. Large age-gap face verification by feature injection in deep networks. Pattern Recognition Letters, 90:36-42, 2017.

4. Deng Cai, Xiaofei He, Yuxiao Hu, and Jiawei Han. Learning a spatially smooth subspace for face recognition. In IEEE Conference on Computer Vision and Pattern Recognition, pages 1-7, 2007.

5. Kuang-Yu Chang, Chu-Song Chen, and Yi-Ping Hung. Ordinal hyperplanes ranker with cost sensitivities for age estimation. In Computer Vision and Pattern Recognition (CVPR), 2011 IEEE Conference on, pages 585592. IEEE, 2011.

6. G Chiachia. Learning person-specific representations from faces in the wild. IEEE Transactions on Information Forensics and Security, 9(12):2089-2099, 2014.

7. Yun Fu, Ye Xu, and Thomas S Huang. Estimating human age by manifold analysis of face pictures and regression on aging features. In Multimedia and Expo, 2007 IEEE International Conference on, pages 13831386. IEEE, 2007.

8. Xin Geng, Chao Yin, and Zhi-Hua Zhou. Facial age estimation by learning from label distributions. IEEE Transactions on Pattern Analysis and Machine Intelligence, 35(10):2401-2412, 2013. 
9. Xin Geng, Zhi-Hua Zhou, and Kate Smith-Miles. Automatic age estimation based on facial aging patterns. Pattern Analysis and Machine Intelligence, IEEE Transactions on, 29(12):2234-2240, 2007.

10. Asuman Gíźnay and Vasif Nabiyev. A new facial age estimation method using centrally overlapped block based local texture features. Multimedia Tools and Applications, (1):1-27, 2017.

11. Guodong Guo, Yun Fu, Charles R Dyer, and Thomas S Huang. Image-based human age estimation by manifold learning and locally adjusted robust regression. Image Processing, IEEE Transactions on, 17(7):1178-1188, 2008.

12. Guodong Guo, Yun Fu, Thomas S Huang, and Charles R Dyer. Locally adjusted robust regression for human age estimation. In Applications of Computer Vision, 2008. WACV 2008. IEEE Workshop on, pages 1-6. IEEE, 2008.

13. Guodong Guo and Guowang Mu. Human age estimation: What is the influence across race and gender? In Computer Vision and Pattern Recognition Workshops, pages 71-78, 2010.

14. Guodong Guo and Guowang Mu. A framework for joint estimation of age, gender and ethnicity on a large database ąî. Image and Vision Computing, 32(10):761770, 2014.

15. Arthur E. Hoerl, Robert W. Kannard, and Kent F. Baldwin. Ridge regression:some simulations. Communications in Statistics, 4(2):105-123, 1975.

16. Nikhil Ketkar. Stochastic Gradient Descent. 2015.

17. Sharad Kohli, Surya Prakash, and Phalguni Gupta. Hierarchical age estimation with dissimilarity-based classification. Neurocomputing, 120:164-176, 2013.

18. Farshad Kooti, Kristina Lerman, Luca Maria Aiello, Mihajlo Grbovic, Nemanja Djuric, and Vladan Radosavljevic. Portrait of an online shopper:understanding and predicting consumer behavior. In Proceedings of The ACM International Conference on Web Search and Data Mining, pages 205-214, 2016.

19. Andreas Lanitis, Chrisina Draganova, and Chris Christodoulou. Comparing different classifiers for automatic age estimation. Systems, Man, and Cybernetics, Part B: Cybernetics, IEEE Transactions on, 34(1):621628, 2004.

20. Andreas Lanitis, Christopher J. Taylor, and Timothy F Cootes. Toward automatic simulation of aging effects on face images. Pattern Analysis and Machine Intelligence, IEEE Transactions on, 24(4):442-455, 2002.

21. Changsheng Li, Qingshan Liu, Jing Liu, and Hanqing Lu. Learning distance metric regression for facial age estimation. In Pattern Recognition (ICPR), 2012 21st International Conference on, pages 2327-2330. IEEE, 2012.
22. Changsheng Li, Qingshan Liu, Jing Liu, and Hanqing Lu. Learning ordinal discriminative features for age estimation. In Computer Vision and Pattern Recognition (CVPR), 2012 IEEE Conference on, pages 2570-2577. IEEE, 2012.

23. Changsheng Li, Qingshan Liu, Jing Liu, and Hanqing Lu. Ordinal distance metric learning for image ranking. IEEE Transactions on Neural Networks and Learning Systems, 26(7):1551, 2015.

24. Hao Liu, Jiwen Lu, Jianjiang Feng, and Jie Zhou. Labelsensitive deep metric learning for facial age estimation. IEEE Transactions on Information Forensics and Security, 13:292-305, 2018.

25. Mingxia Liu, Daoqiang Zhang, Songcan Chen, and Hui Xue. Joint binary classifier learning for ecoc-based multi-class classification. IEEE Transactions on Pattern Analysis and Machine Intelligence, 38(11):2335-2341, 2016.

26. Ziwei Liu, Ping Luo, Xiaogang Wang, and Xiaoou Tang. Deep learning face attributes in the wild. In Proceedings of The IEEE International Conference on Computer Vision, pages 3730-3738, 2015.

27. Khoa Luu, Karl Ricanek, Tien D Bui, and Ching Y Suen. Age estimation using active appearance models and support vector machine regression. In Biometrics: Theory, Applications, and Systems, 2009. BTAS'09. IEEE 3rd International Conference on, pages 1-5. IEEE, 2009.

28. Samuel Marchal, Xiuyan Jiang, Radu State, and Thomas Engel. A big data architecture for large scale security monitoring. In Proceedings of The IEEE International Congress on Big Data, pages 56-63, 2014.

29. Lukas Meier, Sara Van De Geer, and Peter Bĺźhlmann. The group lasso for logistic regression. Journal of the Royal Statistical Society, 70(1):53-71, 2008.

30. Stylianos Moschoglou, Athanasios Papaioannou, Christos Sagonas, Jiankang Deng, Irene Kotsia, and Stefanos Zafeiriou. Agedb: The first manually collected, in-thewild age database. In International Conference on Computer Vision and Pattern Recognition Workshops, pages 1997-2005, 2017.

31. Zhenxing Niu, Mo Zhou, Le Wang, Xinbo Gao, and Gang Hua. Ordinal regression with multiple output c$\mathrm{nn}$ for age estimation. In Computer Vision and Pattern Recognition, pages 4920-4928, 2016.

32. Lianyong Qi, Ruili Wang, Shancang Li, Qiang He, Xiaolong $\mathrm{Xu} \mathrm{Xu}$, and Chunhua $\mathrm{Hu}$. Time-aware distributed service recommendation with privacy-preservation. Information Sciences, 480:354-364, 2019.

33. Lianyong Qi, Xuyun Zhang, Wanchun Dou, and Qiang Ni. A distributed locality-sensitive hashing-based approach for cloud service recommendation from multisource data. IEEE Journal on Selected Areas in Com- 
munications, 35(11):2616-2624, 2017.

34. Lianyong Qi, Zhili Zhou, Jiguo Yu, and Qi Liu. Datasparsity tolerant web service recommendation approach based on improved collaborative filtering. IEICE Transactions on Information and Systems, E100.D(9):20922099, 2017.

35. Karl Ricanek and Tamirat Tesafaye. Morph: a longitudinal image database of normal adult age-progression. In International Conference on Automatic Face and Gesture Recognition, pages 341-345, 2006.

36. Phyo-Kyaw Sai, Jian-Gang Wang, and Eam-Khwang Teoh. Facial age range estimation with extreme learning machines. Neurocomputing, 149:364-372, 2015.

37. Catherine M. Scandrett, Christopher J. Solomon, and Stuart J. Gibson. A person-specific, rigorous aging model of the human face. Pattern Recognition Letters, 27(15):1776-1787, 2006.

38. Karen Simonyan and Andrew Zisserman. Very deep convolutional networks for large-scale image recognition. In International Conference on Learning Representations, pages 1-14, 2015.

39. Qing Tian, Meng Cao, and Tinghuai Ma. Feature relationships learning incorporated age estimation assisted by cumulative attribute encoding. Computers, Materials and Continua, 56(3):467-482, 2018.

40. Qing Tian and Songcan Chen. Cross-heterogeneousdatabase age estimation through correlation representation learning. Neurocomputing, 238:286-295, 2017.

41. Qing Tian, Songcan Chen, and Xiaoyang Tan. Comparative study among three strategies of incorporating spatial structures to ordinal image regression. Neurocomputing, 136(8):152-161, 2014.

42. Qing Tian, Hui Xue, and Lishan Qiao. Human age estimation by considering both the ordinality and similarity of ages. Neural Processing Letters, 43(2):505-521, 2016.

43. Kazuya Ueki, Teruhide Hayashida, and Tetsunori Kobayashi. Subspace-based age-group classification using facial images under various lighting conditions. In Automatic Face and Gesture Recognition, 2006. FGR 2006. 7th International Conference on, pages 6-pp. IEEE, 2006.
44. Andreas Wachter and Lorenz T. Biegler. On the implementation of an interior-point filter line-search algorith$\mathrm{m}$ for large-scale nonlinear programming. Mathematical Programming, 106(1):25-57, 2006.

45. Xuefeng Xi, Victor S. Sheng, Binqi Sun, Lei Wang, and Fuyuan Hu. An empirical comparison on multi-target regression learning. Computers, Materials and Continua, 56(2):185-198, 2018.

46. Jiucheng Xie and Chiman Pun. Chronological age estimation under the guidance of age-related facial attributes. IEEE Transactions on Information Forensics and Security, 14(9):2500-2511, 2019.

47. Junliang Xing, Kai Li, Weiming Hu, Chunfeng Yuan, and Haibin Ling. Diagnosing deep learning models for high accuracy age estimation from a single image. Pattern Recognition, 66:106-116, 2017.

48. Shuicheng Yan, Huan Wang, Thomas S Huang, Qiong Yang, and Xiaoou Tang. Ranking with uncertain labels. In Multimedia and Expo, 2007 IEEE International Conference on, pages 96-99. IEEE, 2007.

49. Shuicheng Yan, Huan Wang, Xiaoou Tang, and Thomas S Huang. Learning auto-structured regressor from uncertain nonnegative labels. In Computer Vision, 2007. ICCV 2007. IEEE 11th International Conference on, pages 1-8. IEEE, 2007.

50. Xu Yang, Bin Bin Gao, Chao Xing, and Zeng Wei Huo. Deep label distribution learning for apparent age estimation. In IEEE International Conference on Computer Vision Workshop, pages 344-350, 2016.

51. Yu Zhang and Dit Yan Yeung. A convex formulation for learning task relationships in multi-task learning. In The Conference on Uncertainty in Artificial Intelligence, pages 733-742, 2010.

52. Yuhong Zhang, Qinqin Wang, Yuling Li, and Xindong Wu. Sentiment classification based on piecewise pooling convolutional neural network. Computers, Materials and Continua, 56(2):285-297, 2018.

53. Zhili Zhou, Yunlong Wang, Q. M. Jonathan Wu, Ching Nung Yang, and Xingming Sun. Effective and efficient global context verification for image copy detection. IEEE Transactions on Information Forensics and Security, 12(1):48-63, 2017. 